\title{
Phonon traps reduce the quasiparticle density in superconducting circuits
}

Cite as: Appl. Phys. Lett. 115, 212601 (2019); https://doi.org/10.1063/1.5124967

Submitted: 20 August 2019. Accepted: 04 November 2019. Published Online: 21 November 2019

Fabio Henriques, Francesco Valenti (D), Thibault Charpentier, Marc Lagoin, Clement Gouriou (D), Maria Martínez (D), Laura Cardani (D), Marco Vignati (D), Lukas Grünhaupt (D), Daria Gusenkova, Julian Ferrero, Sebastian T. Skacel (D), Wolfgang Wernsdorfer (D), Alexey V. Ustinov, Gianluigi Catelani $\mathbb{D}^{\mathrm{D}}$, Oliver Sander, and loan M. Pop (D)

Export citation

\section{ARTICLES YOU MAY BE INTERESTED IN}

A quantum engineer's guide to superconducting qubits

Applied Physics Reviews 6, 021318 (2019); https://doi.org/10.1063/1.5089550

Electromechanical behavior of graphene foams

Applied Physics Letters 115, 211902 (2019); https://doi.org/10.1063/1.5120446

Carbon nanotube-based lossy transmission line filter for superconducting qubit

measurements

Applied Physics Letters 115, 213504 (2019); https://doi.org/10.1063/1.5116109

Lock-in Amplifiers Find out more today

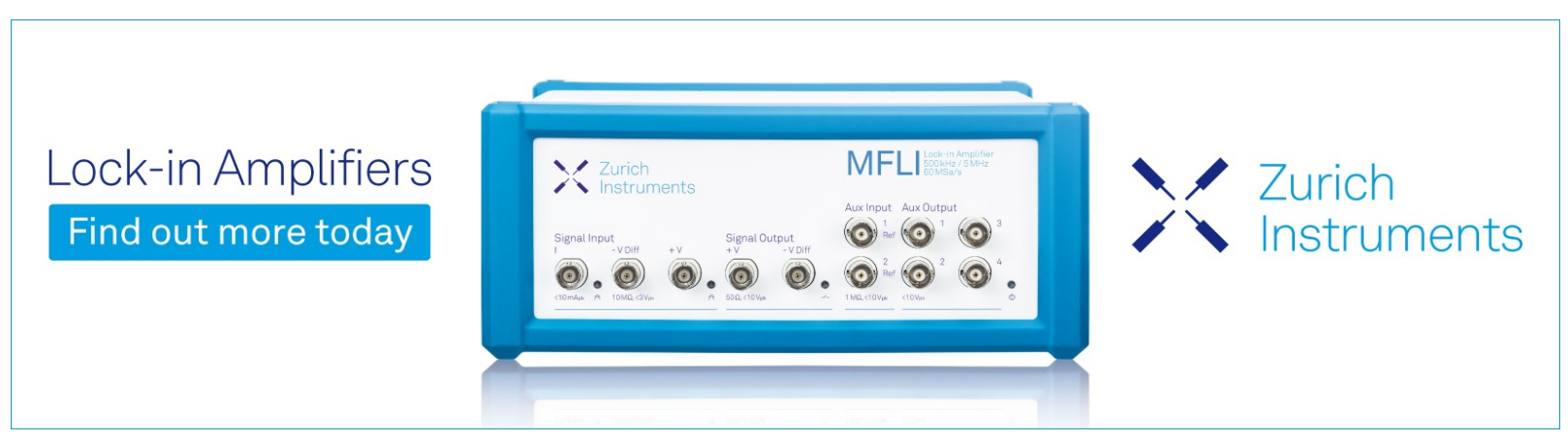

Zurich Instruments 


\title{
Phonon traps reduce the quasiparticle density in superconducting circuits
}

\author{
Cite as: Appl. Phys. Lett. 115, 212601 (2019); doi: 10.1063/1.5124967 \\ Submitted: 20 August 2019 • Accepted: 4 November 2019 . \\ Published Online: 21 November 2019
}

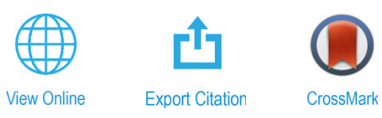

Fabio Henriques, ${ }^{1, a)}$ Francesco Valenti, ${ }^{1,2, a)}$ (D) Thibault Charpentier, ${ }^{1}$ Marc Lagoin, ${ }^{7}$ Clement Gouriou, ${ }^{1}$ Maria Martínez, ${ }^{3}$ (D) Laura Cardani, ${ }_{4}^{4}$ (D) Marco Vignati, ${ }^{4}$ (D) Lukas Grünhaupt, ${ }^{7}$ (D) Daria Gusenkova, Julian Ferrero, Sebastian T. Skacel, ${ }^{1}$ (iD Wolfgang Wernsdorfer, ${ }^{1,5}$ (iD) Alexey V. Ustinov, ${ }^{1,6}$ Gianluigi Catelani, ${ }^{7}$ (iD Oliver Sander, ${ }^{2}$ and loan M. Pop ${ }^{1,5, b)}$ iD

\author{
AFFILIATIONS \\ ${ }^{7} \mathrm{PHI}$, Karlsruhe Institute of Technology, 76131 Karlsruhe, Germany \\ ${ }^{2}$ IPE, Karlsruhe Institute of Technology, 76344 Eggenstein-Leopoldshafen, Germany \\ ${ }^{3}$ LFNAE, Universidad de Zaragoza, 50009 Zaragoza, Spain \\ ${ }^{4}$ INFN-Sezione di Roma, Piazzale Aldo Moro 2, 00185 Roma, Italy \\ ${ }^{5}$ INT, Karlsruhe Institute of Technology, 76344 Eggenstein-Leopoldshafen, Germany \\ ${ }^{6}$ RQC, National University of Science and Technology MISIS, 119049 Moscow, Russia \\ 7JARA Institute for Quantum Information (PGI-11), Forschungszentrum Jülich, 52425 Jülich, Germany

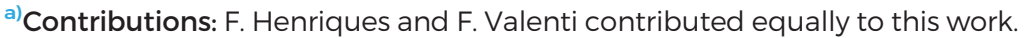

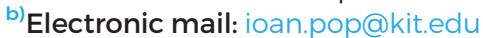

\begin{abstract}
Out of equilibrium quasiparticles (QPs) are one of the main sources of decoherence in superconducting quantum circuits and one that is particularly detrimental in devices with high kinetic inductance, such as high impedance resonators, qubits, and detectors. Despite significant progress in the understanding of QP dynamics, pinpointing their origin and decreasing their density remain outstanding tasks. The cyclic process of recombination and generation of QPs implies the exchange of phonons between the superconducting thin film and the underlying substrate. Reducing the number of substrate phonons with frequencies exceeding the spectral gap of the superconductor should result in a reduction of QPs. Indeed, we demonstrate that surrounding high impedance resonators made of granular aluminum (grAl) with lower gapped thin film aluminum islands increases the internal quality factors of the resonators in the single photon regime, suppresses the noise, and reduces the rate of observed QP bursts. The aluminum islands are positioned far enough from the resonators to be electromagnetically decoupled, thus not changing the resonator frequency nor the loading. We therefore attribute the improvements observed in grAl resonators to phonon trapping at frequencies close to the spectral gap of aluminum, well below the grAl gap.
\end{abstract}

Published under license by AIP Publishing. https://doi.org/10.1063/1.5124967

Superconducting circuits play a central role in research and application areas such as solid state quantum optics, ${ }^{1}$ metrology, ${ }^{2,3}$ and low temperature detectors. ${ }^{4,5}$ In particular, the field of superconducting qubits has grown impressively during the last decade. ${ }^{6,7}$ In these devices, quantum states can live for up to tens of microseconds, while gate times can be as short as tens of nanoseconds. ${ }^{8-11}$ Nevertheless, coherence times need to be further improved by orders of magnitude in order to be able to perform quantum error correction ${ }^{12,13}$ with an affordable hardware overhead.

One of the main sources of decoherence in superconducting devices at millikelvin temperatures are out of equilibrium quasiparticles (QPs), ${ }^{14-22}$ which can be viewed as broken Cooper pairs (CPs).
Quasiparticles can be particularly damaging in high kinetic inductance circuits, ${ }^{23-27}$ which are a promising avenue for protected qubits ${ }^{28}$ and hybrid superconducting-semiconducting devices. ${ }^{29-31}$ Proposed mechanisms for CP breaking include stray infrared radiation, ${ }^{32,33}$ direct microwave drive, ${ }^{34,35}$ and high energy phonons in the device substrate created by environmental or cosmic radioactivity. ${ }^{36-38}$ The latter is particularly damaging because it gives rise to correlated QP bursts in multiple devices on the same chip, ${ }^{36,39}$ possibly resulting in correlated errors, further complicating error correction.

Quasiparticle mitigation strategies can be divided into two categories: Removing QPs, e.g., by trapping them in normal metals, ${ }^{40,41}$ vortices, ${ }^{20,21,42}$ and pumping them outside the susceptible region of 
the circuit, ${ }^{25}$ or preventing CP breaking, e.g., by filtering and shielding from radiation with a frequency above the superconducting gap. ${ }^{32}$

Here, we describe a complementary method of preventing $\mathrm{CP}$ breaking by reducing high energy substrate phonons. We show that the figures of merit of superconducting grAl resonators, such as single photon internal quality factor and noise spectral density, can be improved by surrounding them with lower gapped islands made of pure aluminum, which act as phonon traps.

Our approach is similar to that of Refs. 38 and 43, in which it has recently been demonstrated that surrounding kinetic inductance detectors with a lower gapped superconducting film reduces the number of measurable QP bursts by an order of magnitude and the noise equivalent power by a factor three. Phonon traps downconvert the frequency of high energy substrate phonons to that of their own spectral gap via inelastic electron-phonon interactions. Therefore, phonons resulting from recombination in the traps are unable to break CPs in the circuit.

This phenomenological model is detailed in Ref. 43 , where it is shown that the phonon traps' efficiency increases with the difference between the spectral gaps of the circuit and trap materials. In the following, we demonstrate that the efficiency rapidly scales with the traps' surface. We report more than a factor two improvement in single photon internal quality factors, as well as a reduction of the noise amplitude by an order of magnitude, for traps covering as little as a third of one side of the substrate.

We use superconducting grAl resonators because they can have a kinetic inductance fraction close to unity, thus providing a high susceptibility to QPs, while also retaining high internal quality factors $Q_{i}$ in the range of $10^{4}-10^{5}$ in the single photon regime. ${ }^{26,44,45}$ Granular aluminum is a composite material made of self-assembled $\mathrm{Al}$ grains, $3-4 \mathrm{~nm}$ in diameter, embedded into an amorphous $\mathrm{AlO}_{\mathrm{x}}$ matrix. ${ }^{46,47}$ The oxide thickness is tuned by the oxygen pressure during thin film deposition, yielding resistivities spanning $\rho=1-10^{4} \mu \Omega \mathrm{cm}$. Thanks to the corresponding kinetic inductances up to the $\mathrm{nH} / \square$ range, grAl has recently attracted interest as a material for high impedance quantum circuits. ${ }^{27}$ The superconducting gap of grAl is dome-shaped as a function of resistivity; ${ }^{48,49}$ for films grown on substrates at room temperature, the critical temperature has a maximum $T_{c, \max } \approx 2.1 \mathrm{~K}$ for $\rho \approx 400 \mu \Omega \mathrm{cm},{ }^{49}$ significantly above the critical temperature of thin film aluminum, $T_{c, \mathrm{Al}} \approx 1.4 \mathrm{~K}$. The resulting difference in the spectral gaps allows $\mathrm{Al}$ to be used as a phonon trap for grAl circuits. ${ }^{43}$

We fabricate all resonators in one step using an optical lift-off and electron beam evaporation of a $20 \mathrm{~nm}$ thick grAl film on a $330 \mu \mathrm{m}$ thick, double-side polished $c$-plane sapphire wafer. We employ a grAl film with $\rho=5 \mathrm{~m} \Omega \mathrm{cm}$ and $T_{c} \approx 1.8 \mathrm{~K}$ in order to maximize the sensitivity to QP bursts, while remaining a factor two below the edge of the superconductor-to-insulator transition. ${ }^{49}$ The resulting kinetic inductance per square is $L_{K}=2 \mathrm{nH} / \square$, orders of magnitude larger than the geometric inductance.

As shown in Fig. 1, and similar to Ref. 26, each chip hosts three grAl resonators with sizes of $600 \times 10,1000 \times 40$, and $420 \times 5 \mu \mathrm{m}^{2}$, which we label A, B, and C, respectively. The resonators are surrounded by a square lattice of $10 \times 10 \mu \mathrm{m}^{2}$ aluminum islands, $60 \mathrm{~nm}$ thick, deposited in a second lithographic step, using the same lift-off technique employed for the resonators. We fabricate three chips with various lattice parameters, $d=20,10$, and $5 \mu \mathrm{m}$, in order to achieve an increasingly larger phonon trap filling factor $(F)$ of $8.5 \%, 19 \%$, and $34 \%$, respectively. We also fabricate a witness chip without traps $(F=0)$.

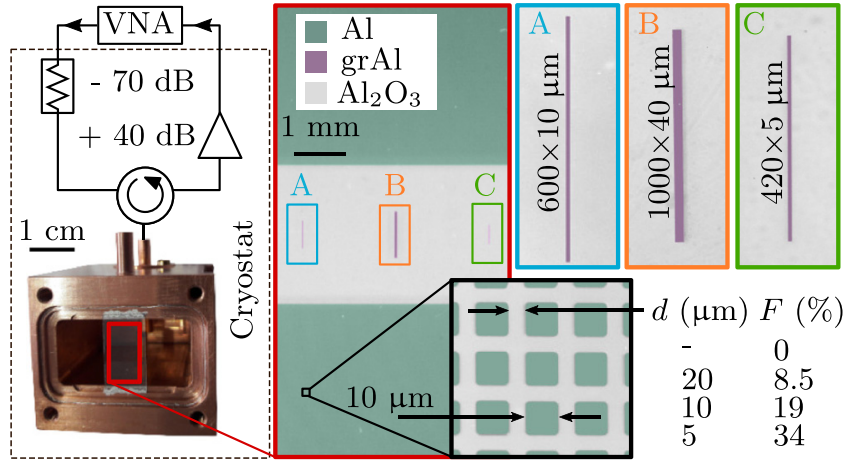

FIG. 1. Photograph of a Cu waveguide housing a $15 \times 8 \times 0.33 \mathrm{~mm}^{3}$ sapphire chip (red box) supporting three $20 \mathrm{~nm}$ thick grAl resonators (highlighted in magenta, labeled $\mathrm{A}-\mathrm{C}$ ) and a square lattice of $60 \mathrm{~nm}$ thick Al phonon traps (jade-colored squares outlined in the black box), all patterned via an optical lift-off lithography. We sweep the spacing $d$ between $\mathrm{Al}$ islands to obtain different phonon trap filling factors $F$, defined as the fraction of the substrate covered by traps and listed in the inset table. The chip is glued to the waveguide using silver paste. The waveguide, anchored to the mixing chamber of a dilution cryostat at $15 \mathrm{mK}$, is connected to a reduced schematics of the microwave reflection setup (cf. supplementary material).

Using the phonon trapping model of Ref. 43, based on Refs. $50-53$, for grAl resonators in the presence of $\mathrm{Al}$ islands (cf. supplementary material), we show that the internal dissipation rate $1 / Q_{i}$ and QP burst rate $\Gamma_{B}$ decrease as a function of increasing $F$

$$
\begin{gathered}
Q_{i}^{-1}=Q_{\mathrm{QP}, 0}^{-1} \sqrt{1+(\beta F)^{2}-\beta F \sqrt{2+(\beta F)^{2}}}+p \tan \delta, \\
\Gamma_{B}=\Gamma_{0} \Lambda /(F+\Lambda) .
\end{gathered}
$$

Here, $Q_{\mathrm{QP}, 0}$ and $\Gamma_{0}$ are the $\mathrm{QP}$-dependent internal quality factor and QP burst rate for $F=0$, respectively, $\tan \delta$ is the dielectric loss tangent, and $p=1.5 \times 10^{-4}$ is the dielectric participation ratio of the resonators. ${ }^{26}$ The coefficient $\beta$ is a phenomenological constant which accounts for the rates of phonon generation, scattering, and thermalization, and $\Lambda$ is the ratio between the rates of phonon thermalization to the sample holder and phonon absorption in the traps.

We would like to note that both the island size and the lattice parameter $d$ are two orders of magnitude larger than the wavelength of phonons resulting from $\mathrm{QP}$ recombination in grAl and $\mathrm{Al}$, which is in the range of $50-100 \mathrm{~nm}$, considering a speed of sound in the sapphire of about $10 \mathrm{~km} / \mathrm{s}^{54}$ The propagation of phonons in the substrate is thus unhindered by gaps in the phonon dispersion relation. However, phononic crystal engineering could also be a viable phonon mitigation approach, as demonstrated by the shielding of optomechanical resonators from phonons at gigahertz frequencies. ${ }^{5}$

The sapphire chip is glued to the $\mathrm{Cu}$ waveguide shown in Fig. 1 using silver paste. We use thin indium wire to ensure both tight sealing and electrical contact between the waveguide and its cap (not shown). The resonators couple to the TE10 waveguide mode, providing a low loss microwave environment. ${ }^{56}$ The waveguide is placed into successive thermal and magnetic shields, and the microwave lines are heavily attenuated and shielded, similar to the setup of Ref. 26.

We measure the complex reflection coefficient $\Gamma$ of the grAl resonators using a vector network analyzer (VNA). In Fig. 2(a), we plot the typical measurement results of $\Gamma$ vs frequency in the complex 
plane for two resonators of type A, with different filling factors, $F=0$ and $\mathrm{F}=19 \%$. The larger diameter of the reflection circle for the resonator surrounded by phonon traps indicates reduced losses.

In Fig. 2(b), we plot the fitted internal quality factors $Q_{i}$ as a function of the phonon trap filling factor for all resonators. We observe an overall trend of $Q_{i}$ increasing with $F$, which can be fitted to Eq. (1) by choosing $\beta=9$ for all resonators (cf. supplementary material). This allows us to extrapolate that going from $F=0$ to $F \rightarrow 1$, the single photon $Q_{i}$ can be, in principle, increased by up to an order of magnitude. The extracted $\langle\tan \delta\rangle=6.6 \times 10^{-3}$ for resonators $\mathrm{A}$ and $\mathrm{B}$ is (a)

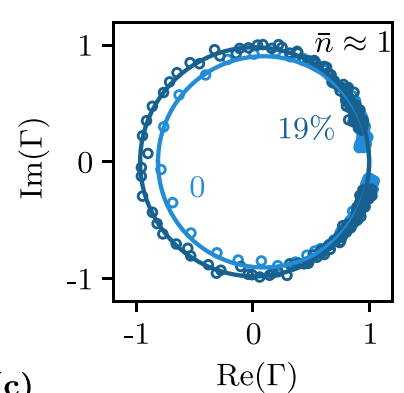

(c)

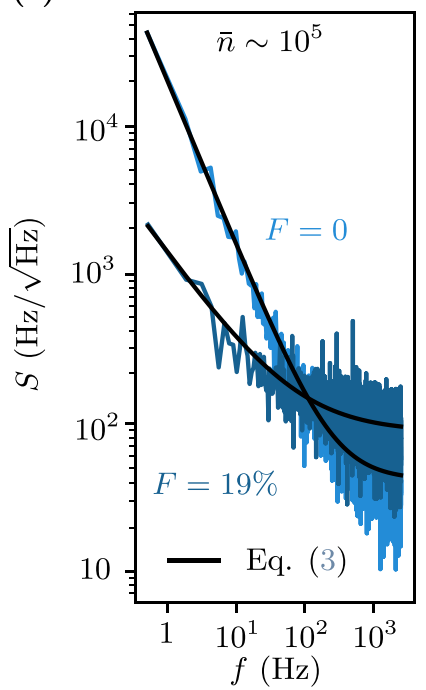

(b)

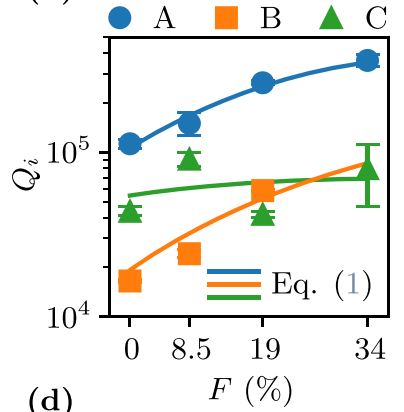

(d)
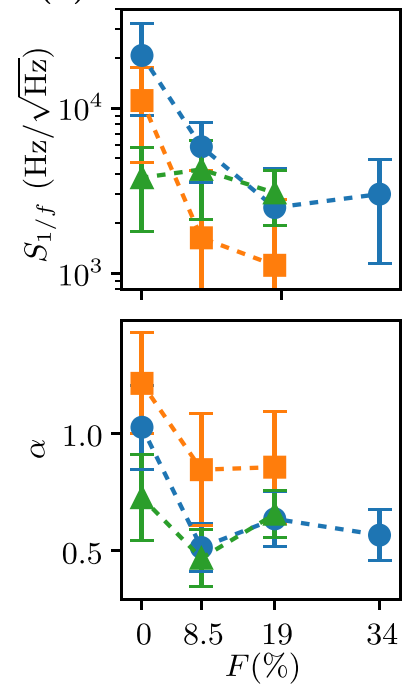

FIG. 2. Effect of phonon trapping on resonator dissipation and noise. (a) Typical measurement of the real and imaginary components of the reflection coefficient $\Gamma$, normalized to the waveguide response, for two resonators $A$ with different filling factors $F=0$ (light blue) and $F=19 \%$ (blue), in the single photon regime. (b) Internal quality factors in the single photon regime extracted from the circle fit (cf. supplementary material), plotted as a function of the filling factor. Error bars represent the fitting routine uncertainty. The solid lines are fits to Eq. (1). (c) Noise spectral density $S(f)$ of the two resonators shown in panel (a). We compute the spectra from time traces, each four seconds long, in which no QP bursts are present [in contrast with the time traces shown in Fig. 3(a)]. We fit the data (black lines) with the phenomenological model of Eq. (3). (d) Fitted noise amplitude $S_{1 / f}$ (top panel) and exponent $\alpha$ (bottom panel) plotted as a function of the filling factor. We obtain the plotted values by averaging over tens of spectra. For resonator $\mathrm{C}$ with $F=34 \%$, we cannot measure the $1 / f$ spectrum because its frequency lies outside the measurement band, limited at $8 \mathrm{GHz}$. Error bars represent one standard deviation. The dashed lines connecting the markers are guides to the eye. comparable to the values reported in Refs. 26 and 57. For resonators $\mathrm{C},\langle\tan \delta\rangle=10^{-1}$, hinting at an increased dielectric loss at least for some of those resonators, possibly due to an optical photoresist residue at the interface. ${ }^{58}$

The measurements of the internal quality factors presented in Figs. 2(a) and 2(b) were obtained using a readout drive corresponding to a circulating photon number $\bar{n} \approx 1$. The photon number is calibrated using the formula $\bar{n}=4 P_{\text {cold }} Q_{\text {tot }}^{2} / \hbar \omega_{0}^{2} Q_{c}$, where $P_{\text {cold }}$ is the onsample drive, $Q_{\text {tot }}^{-1}=Q_{i}^{-1}+Q_{c}^{-1}$, and $Q_{c}$ is the coupling quality factor. At stronger drives $(\bar{n} \gg 1), Q_{i}$ is further increased, either by saturating dielectric loss ${ }^{59,60}$ or by enhancing QP diffusion; ${ }^{25,61}$ however, it is also less correlated with $F$ (cf. supplementary material), possibly due to the onset of a more complex QP and phonon dynamics which remains to be understood.

Resonant frequencies and coupling quality factors for all resonators are summarized in the supplementary material. Resonator B with $\mathrm{F}=34 \%$ could not be measured, most likely due to its resonant frequency being outside of the frequency band of the setup.

In Fig. 2(c), we plot the noise spectral density $S(f)$ for resonator A with filling factors $F=0$ and $19 \%$ measured at the highest power before bifurcation $\left(\bar{n} \sim 10^{5}\right)$. Note the order of magnitude reduction in the low frequency noise amplitude for the sample with phonon traps; the noise floor at a high frequency is given by the readout electronics. Interestingly, the amplitude of the $1 / \mathrm{f}$ noise does not depend on $\bar{n}$ (cf. supplementary material). We fit the noise spectra with the phenomenological model

$$
S(f)=S_{0}+S_{1 / f} /(f / 1 \mathrm{~Hz})^{\alpha} .
$$

In Fig. 2(d), we plot the fitted amplitude $S_{1 / f}$ and exponent $\alpha$ for noise spectra of all measured resonators vs $F$. For resonators A and B, we observe an overall decreasing trend for both the amplitude and the exponent of the noise as a function of the phonon trap filling factor. This trend is consistent with the observed increase in $Q_{i}$ [cf. Fig. 2(b)] and indicates QP generation-recombination as a primary source of noise. ${ }^{18}$ In Fig. 3(a), we show the time evolution of the resonant frequency $f_{0}$ for resonators $\mathrm{A}$ with $F=0$ and $19 \%$. The time traces of the resonant frequency show noise that is qualitatively similar to the one reported in Refs. 18, 26, and 36: Stochastic QP bursts, which abruptly lower the resonant frequency and are followed by a relaxation tail, interspersed on top of a background of fluctuations. The resonator surrounded by phonon traps with $F=19 \%$ shows a reduction in both the fluctuations and the number of measured QP bursts, indicating a significant reduction in nonequilibrium phonons with energies above the spectral gap of grAl. The QP relaxation after a burst [cf. right hand panels of Fig. 3(a)] is unaffected by F. We fit the exponential tails with the same methodology of Ref. 26 and obtain the QP lifetime $\tau_{\mathrm{qp}}$ $=0.5 \pm 0.1 \mathrm{~s}$ for all resonators and $F$ values.

We measure a constant QP burst rate over the course of several days (cf. supplementary material). We quantify the change in QP density $\delta x_{\mathrm{QP}}=-4 \delta f_{0} / f_{0}$, and we count as bursts all QP generation events above the threshold $\delta x_{\mathrm{QP}, \min }=5 \times 10^{-5}$. The only exception to this method is resonator $C$ with $F=34 \%$, which lies outside the optimal readout band and thus has a higher white noise level, masking small events. For this resonator, the threshold is $\delta x_{\mathrm{QP}, \min }=15 \times 10^{-5}$. In Fig. 3(b), we show that the rate of bursts decreases monotonically with $F$ for all resonators. We interpret this as a decrease in the probability that pair-breaking phonons reach the resonators; the larger the filling 

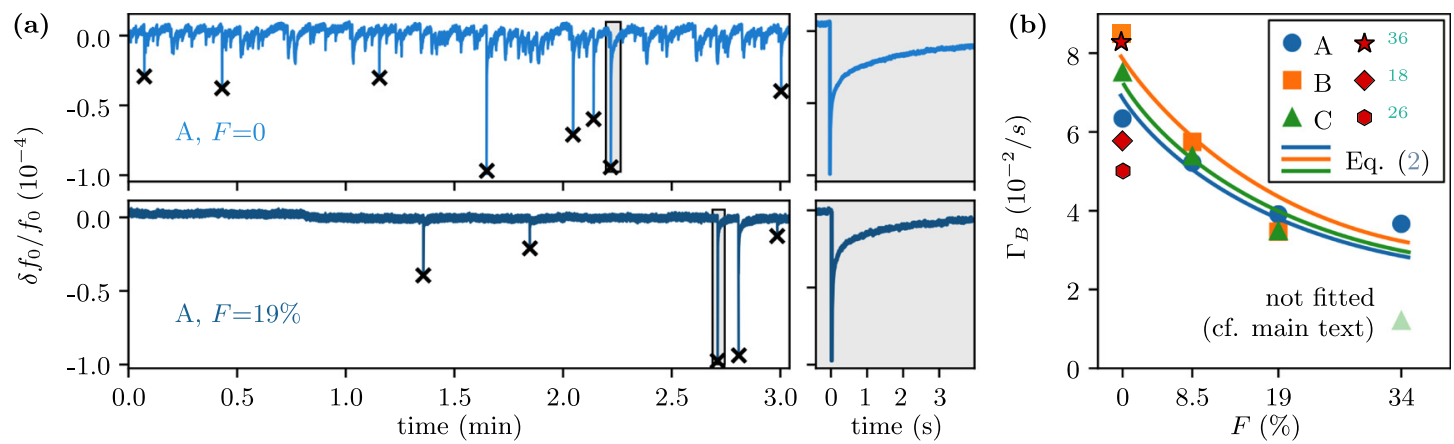

FIG. 3. Effect of phonon trapping on QP bursts. (a) Typical time trace measurement of $\delta f_{0} / f_{0}$ for resonators $A$, filling factor $F=0$ (top panel, light blue), and $F=19 \%$ (bottom panel, blue), with zoom-ins on single QP bursts. Quasiparticle bursts (see the main text for a discussion on their possible origins) are marked with a black cross. Increasing the filling factor $F$ yields a twofold improvement, reducing both the low frequency baseline noise [cf. Figs. 2(c) and 2(d)] and the number of observed bursts. In the right hand panels, we plot the typical frequency evolution during the first few seconds after a typical QP burst (black boxes in the respective left hand panel). The relaxation after the burst consists of an initial steep decay, followed by an exponential tail. Note that increasing the filling factor of the traps does not change the QP relaxation dynamics in the grAl resonators. (b) Measured rate of QP bursts $\Gamma_{B}$ for all resonators and all filling factors, averaged over several hours. Error bars are smaller than the marker size and are thus not shown. The QP burst rate decreases monotonically with the filling factor for all resonators. The solid lines are fits to Eq. (2). Due to its higher white noise level, resonator $C$ with $F=34 \%$ is only sensitive to large QP bursts, resulting in a lower rate, and it is thus omitted from the fit (cf. supplementary material for details). For comparison, we also show the previously reported QP burst rates (red markers) for both $\mathrm{grAl}^{26}$ and $\mathrm{Al}$.

factor, the more effective the phonon trapping. We fit the QP burst rate to Eq. (2) using the phonon relaxation ratio $\Lambda=0.24$ for all resonators (cf. supplementary material), which shows that the QP burst rate can, in principle, be reduced by a factor $(1+\Lambda) / \Lambda \sim 5$ for $F \rightarrow 1$. The rate difference between the resonator types may be due to their position with respect to the standing wave patterns of the substrate phonons. ${ }^{62}$

Interestingly, for similarly sized substrates without phonon traps, the measured QP burst rates are comparable [cf. Fig. 3(b) and Refs. 18, 26, and 36]. The impact rate of cosmic muons on the substrate can account for up to $30 \%$ of the measured rate, ${ }^{63}$ with the rest possibly originating from various environmental radioactive sources, which should be further investigated.

In summary, we measured 11 grAl resonators with resistivity $\rho \approx 5 \mathrm{~m} \Omega \mathrm{cm}$, corresponding to a kinetic inductance of $2 \mathrm{nH} / \square$. Out of these, 8 were fabricated on chips containing $\mathrm{Al}$ islands with varying filling factors. The $\mathrm{Al}$ islands are electromagnetically decoupled from the resonators and act as phonon traps due to their lower superconducting gap compared to grAl. When increasing the phonon trap density, we observe three types of improvement of resonator performance: Single photon internal quality factors increase by up to a factor three, the $1 / \mathrm{f}$ noise is reduced by an order of magnitude, and the rate of QP bursts is halved. These results indicate that nonthermal phonons in the substrate play an important role in the generation of nonequilibrium QPs in the superconducting circuits, and phonon frequency downconversion can be an effective strategy to reduce the QP density.

Future work should focus on maximizing the filling factor $F$ and decreasing the phonon relaxation ratio $\Lambda$ by employing traps with a decreased gap and an increased thickness. Further improvements might be achieved by engineering the phonon dispersion relation in the substrate, by placing the superconducting devices in the regions of lower phonon density, by identifying and removing hot phonon sources, or by improving phonon thermalization, thus reducing the QP burst rate $\Gamma_{0}$.
See the supplementary material for the cryogenic setup, the phonon trapping model of Ref. 43, the power dependence of resonator noise and dissipation, and the QP burst counting method.

We are grateful to A. Monfardini, J. Baselmans, and P. de Visser for insightful discussions and to $\mathrm{L}$. Radtke and A. Lukashenko for the technical support. Funding was provided by the Alexander von Humboldt foundation in the framework of a Sofja Kovalevskaja award endowed by the German Federal Ministry of Education and Research, and by the Initiative and Networking Fund of the Helmholtz Association, within the Helmholtz future project scalable solid state quantum computing. A.V.U. received partial support from the Ministry of Education and Science of the Russian Federation program to increase the competitiveness of the NUST MISIS, Nos. K2-2018-015 and K2-2017-081.

\section{REFERENCES}

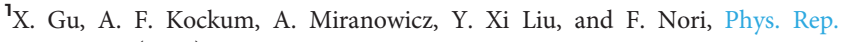
718-719, 1 (2017).

${ }^{2}$ B. Taylor and T. Witt, Metrologia 26, 47 (1989).

${ }^{3}$ J. P. Pekola, O.-P. Saira, V. F. Maisi, A. Kemppinen, M. Möttönen, Y. A. Pashkin, and D. V. Averin, Rev. Mod. Phys. 85, 1421 (2013).

${ }^{4}$ P. K. Day, H. G. LeDuc, B. A. Mazin, A. Vayonakis, and J. Zmuidzinas, Nature 425, 817 (2003).

${ }^{5}$ J. Zmuidzinas, Annu. Rev. Condens. Matter Phys. 3, 169 (2012).

${ }^{6}$ J. M. Gambetta, J. M. Chow, and M. Steffen, npj Quantum Inf. 3, 2 (2017).

${ }^{7}$ P. Krantz, M. Kjaergaard, F. Yan, T. P. Orlando, S. Gustavsson, and W. D. Oliver, Appl. Phys. Rev. 6, 021318 (2019).

${ }^{8}$ F. Yan, S. Gustavsson, A. Kamal, J. Birenbaum, A. P. Sears, D. Hover, T. J. Gudmundsen, D. Rosenberg, G. Samach, S. Weber et al., Nat. Commun. 7, 12964 (2016).

${ }^{9}$ M. A. Rol, C. C. Bultink, T. E. O’Brien, S. R. de Jong, L. S. Theis, X. Fu, F. Luthi, R. F. L. Vermeulen, J. C. de Sterke, A. Bruno et al., Phys. Rev. Appl. 7, 041001 (2017).

${ }^{10}$ P. V. Klimov, J. Kelly, Z. Chen, M. Neeley, A. Megrant, B. Burkett, R. Barends, K. Arya, B. Chiaro, Y. Chen et al., Phys. Rev. Lett. 121, 090502 (2018).

${ }^{11}$ N. T. Bronn, V. P. Adiga, S. B. Olivadese, X. Wu, J. M. Chow, and D. P. Pappas, Quantum Sci. Technol. 3, 024007 (2018). 
${ }^{12}$ A. G. Fowler, A. C. Whiteside, and L. C. L. Hollenberg, Phys. Rev. Lett. 108, 180501 (2012)

${ }^{13}$ N. Ofek, A. Petrenko, R. Heeres, P. Reinhold, Z. Leghtas, B. Vlastakis, Y. Liu, L. Frunzio, S. M. Girvin, L. Jiang et al., Nature 536, 441-445 (2016).

${ }^{14}$ J. Aumentado, M. W. Keller, J. M. Martinis, and M. H. Devoret, Phys. Rev. Lett. 92, 066802 (2004).

${ }^{15}$ R. Barends, J. Baselmans, S. Yates, J. Gao, J. Hovenier, and T. Klapwijk, Phys. Rev. Lett. 100, 257002 (2008)

${ }^{16}$ M. Shaw, R. Lutchyn, P. Delsing, and P. Echternach, Phys. Rev. B 78, 024503 (2008).

${ }^{17} \mathrm{G}$. Catelani, J. Koch, L. Frunzio, R. Schoelkopf, M. H. Devoret, and L. Glazman, Phys. Rev. Lett. 106, 077002 (2011).

${ }^{18}$ P. De Visser, J. Baselmans, P. Diener, S. Yates, A. Endo, and T. Klapwijk, Phys. Rev. Lett. 106, 167004 (2011).

${ }^{19}$ D. Ristè, C. C. Bultink, M. J. Tiggelman, R. N. Schouten, K. W. Lehnert, and L. DiCarlo, Nat. Commun. 4, 1913 (2013).

${ }^{20}$ C. Wang, Y. Y. Gao, I. M. Pop, U. Vool, C. Axline, T. Brecht, R. W. Heeres, L. Frunzio, M. H. Devoret, G. Catelani et al., Nat. Commun. 5, 5836 (2014).

${ }^{21}$ I. Nsanzineza and B. L. T. Plourde, Phys. Rev. Lett. 113, 117002 (2014).

${ }^{22}$ K. Serniak, M. Hays, G. de Lange, S. Diamond, S. Shankar, L. D. Burkhart, L. Frunzio, M. Houzet, and M. H. Devoret, Phys. Rev. Lett. 121, 157701 (2018).

${ }^{23}$ U. Vool, I. M. Pop, K. Sliwa, B. Abdo, C. Wang, T. Brecht, Y. Y. Gao, S. Shankar, M. Hatridge, G. Catelani et al., Phys. Rev. Lett. 113, 247001 (2014).

${ }^{24}$ C. Janvier, L. Tosi, L. Bretheau, Ç. Ö. Girit, M. Stern, P. Bertet, P. Joyez, D. Vion, D. Esteve, M. F. Goffman et al., Science 349, 1199 (2015).

${ }^{25}$ S. Gustavsson, F. Yan, G. Catelani, J. Bylander, A. Kamal, J. Birenbaum, D. Hover, D. Rosenberg, G. Samach, A. P. Sears et al., Science 354, 1573 (2016).

${ }^{26}$ L. Grünhaupt, N. Maleeva, S. T. Skacel, M. Calvo, F. Levy-Bertrand, A. V. Ustinov, H. Rotzinger, A. Monfardini, G. Catelani, and I. M. Pop, Phys. Rev. Lett. 121, 117001 (2018).

${ }^{27}$ L. Grünhaupt, M. Spiecker, D. Gusenkova, N. Maleeva, S. T. Skacel, I. Takmakov, F. Valenti, P. Winkel, H. Rotzinger, W. Wernsdorfer et al., Nat. Mater. 18, 816 (2019).

${ }^{28}$ P. Groszkowski, A. D. Paolo, A. L. Grimsmo, A. Blais, D. I. Schuster, A. A. Houck, and J. Koch, New J. Phys. 20, 043053 (2018).

${ }^{29}$ J. J. Viennot, M. C. Dartiailh, A. Cottet, and T. Kontos, Science 349, 408 (2015).

${ }^{30}$ X. Mi, J. V. Cady, D. M. Zajac, P. W. Deelman, and J. R. Petta, Science 355, 156 (2017).

${ }^{31}$ A. J. Landig, J. V. Koski, P. Scarlino, U. C. Mendes, A. Blais, C. Reichl, W. Wegscheider, A. Wallraff, K. Ensslin, and T. Ihn, Nature 560, 179 (2018).

${ }^{32}$ R. Barends, J. Wenner, M. Lenander, Y. Chen, R. C. Bialczak, J. Kelly, E. Lucero, P. O’Malley, M. Mariantoni, D. Sank et al., Appl. Phys. Lett. 99, 113507 (2011).

${ }^{33}$ M. Houzet, K. Serniak, G. Catelani, M. Devoret, and L. Glazman, Phys. Rev. Lett. 123, 107704 (2019).

${ }^{34} \mathrm{P}$. De Visser, D. Goldie, P. Diener, S. Withington, J. Baselmans, and T. Klapwijk, Phys. Rev. Lett. 112, 047004 (2014).

${ }^{35}$ U. Patel, I. V. Pechenezhskiy, B. L. T. Plourde, M. G. Vavilov, and R. McDermott, Phys. Rev. B 96, 220501 (2017).

${ }^{36}$ L. Swenson, A. Cruciani, A. Benoit, M. Roesch, C. Yung, A. Bideaud, and A. Monfardini, Appl. Phys. Lett. 96, 263511 (2010).

${ }^{37}$ A. Bespalov, M. Houzet, J. S. Meyer, and Y. V. Nazarov, Phys. Rev. Lett. 117, 117002 (2016).
${ }^{38}$ K. Karatsu, A. Endo, J. Bueno, P. J. de Visser, R. Barends, D. J. Thoen, V. Murugesan, N. Tomita, and J. J. A. Baselmans, Appl. Phys. Lett. 114, 032601 (2019).

${ }^{39}$ D. Moore, S. Golwala, B. Bumble, B. Cornell, P. Day, H. LeDuc, and J. Zmuidzinas, Appl. Phys. Lett. 100, 232601 (2012).

${ }^{40}$ R.-P. Riwar, A. Hosseinkhani, L. D. Burkhart, Y. Y. Gao, R. J. Schoelkopf, L. I. Glazman, and G. Catelani, Phys. Rev. B 94, 104516 (2016).

${ }^{41}$ A. Hosseinkhani, R.-P. Riwar, R. J. Schoelkopf, L. I. Glazman, and G. Catelani, Phys. Rev. Appl. 8, 064028 (2017).

${ }^{42}$ M. Taupin, I. M. Khaymovich, M. Meschke, A. S. Mel'nikov, and J. P. Pekola, Nat. Commun. 7, 10977 (2016).

${ }^{43}$ F. Valenti, F. Henriques, G. Catelani, N. Maleeva, L. Grünhaupt, U. von Lüpke, S. T. Skacel, P. Winkel, A. Bilmes, A. V. Ustinov et al., Phys. Rev. Appl. 11, 054087 (2019).

${ }^{44}$ H. Rotzinger, S. Skacel, M. Pfirrmann, J. Voss, J. Münzberg, S. Probst, P. Bushev, M. Weides, A. Ustinov, and J. Mooij, Supercond. Sci. Technol. 30, 025002 (2017).

${ }^{45}$ W. Zhang, K. Kalashnikov, W.-S. Lu, P. Kamenov, T. DiNapoli, and M. Gershenson, Phys. Rev. Appl. 11, 011003 (2019).

${ }^{46}$ R. W. Cohen and B. Abeles, Phys. Rev. 168, 444 (1968).

${ }^{47}$ G. Deutscher, M. Gershenson, E. Grünbaum, and Y. Imry, J. Vac. Sci. Technol. 10, 697 (1973).

${ }^{48}$ U. S. Pracht, N. Bachar, L. Benfatto, G. Deutscher, E. Farber, M. Dressel, and M. Scheffler, Phys. Rev. B 93, 100503 (2016).

${ }^{49}$ F. Levy-Bertrand, T. Klein, T. Grenet, O. Dupré, A. Benoît, A. Bideaud, O. Bourrion, M. Calvo, A. Catalano, A. Gomez et al., Phys. Rev. B 99, 094506 (2019).

${ }^{50}$ A. Rothwarf and B. N. Taylor, Phys. Rev. Lett. 19, 27 (1967).

${ }^{51}$ S. B. Kaplan, C. C. Chi, D. N. Langenberg, J. J. Chang, S. Jafarey, and D. J. Scalapino, Phys. Rev. B 14, 4854 (1976).

${ }^{52}$ A. V. Sergeev and M. Yu. Reizer, Int. J. Mod. Phys. B 10, 635 (1996).

${ }^{53}$ A. V. Sergeev, V. V. Mitin, and B. S. Karasik, Appl. Phys. Lett. 80, 817 (2002).

${ }^{54}$ J. Winey, Y. Gupta, and D. Hare, J. Appl. Phys. 90, 3109 (2001).

${ }^{55}$ J. Chan, T. P. M. Alegre, A. H. Safavi-Naeini, J. T. Hill, A. Krause, S. Gröblacher, M. Aspelmeyer, and O. Painter, Nature 478, 89-92 (2011).

${ }^{56}$ A. Kou, W. Smith, U. Vool, I. Pop, K. Sliwa, M. Hatridge, L. Frunzio, and M. Devoret, Phys. Rev. Appl. 9, 064022 (2018).

${ }^{57}$ C. Wang, C. Axline, Y. Y. Gao, T. Brecht, Y. Chu, L. Frunzio, M. H. Devoret, and R. J. Schoelkopf, Appl. Phys. Lett. 107, 162601 (2015).

${ }^{58}$ C. M. Quintana, A. Megrant, Z. Chen, A. Dunsworth, B. Chiaro, R. Barends, B. Campbell, Y. Chen, I.-C. Hoi, E. Jeffrey et al., Appl. Phys. Lett. 105, 062601 (2014).

${ }^{59}$ S. Hunklinger, W. Arnold, S. Stein, R. Nava, and K. Dransfeld, Phys. Lett. A 42, 253 (1972).

${ }^{60}$ B. Golding, J. Graebner, B. Halperin, and R. Schutz, Phys. Rev. Lett. 30, 223 (1973).

${ }^{61}$ E. Levenson-Falk, F. Kos, R. Vijay, L. Glazman, and I. Siddiqi, Phys. Rev. Lett. 112, 047002 (2014).

${ }^{62}$ M. Martinez, L. Cardani, N. Casali, A. Cruciani, G. Pettinari, and M. Vignati, Phys. Rev. Appl. 11, 064025 (2019).

${ }^{63}$ R. M. Barnett, C. D. Carone, D. E. Groom, T. G. Trippe, C. G. Wohl, B. Armstrong, P. S. Gee, G. S. Wagman, F. James, M. Mangano et al., Phys. Rev. D 54, 1 (1996). 